\title{
PRODUCTIVIDAD Y COBERTURA DE CULTIVARES DE ALFALFA (Medicago sativa L.) DE DIFERENTES GRADOS DE REPOSO INVERNAL BAJO DOS FRECUENCIAS DE CORTE
}

\author{
Ordoñez, V. N. ${ }^{1} ;$ Nescier, I. de los M. ${ }^{1} ;$ Dovis, V. L. ${ }^{2} ;$ GIECO, J. O. ${ }^{1}$ \\ Bonvin, C. ${ }^{1}$; RIBERO, G. G. ${ }^{1}$ \& WALKer, G. A. ${ }^{1}$
}

\begin{abstract}
RESUMEN
El objetivo de este trabajo fue evaluar la producción de forraje y porcentaje de cobertura de 3 cultivares de alfalfa con distinto grado de reposo invernal (GRI), sometidas a dos frecuencias de corte durante un año. Se utilizaron tres cultivares (GR6-Verzy), (GR9-Mecha) y (GR10-Ruano). El diseño experimental fue de parcelas divididas con cuatro repeticiones. Se establecieron 2 tratamientos definidos por los intervalos entre cortes: T1: 25 días y T2: 35 días. Estos fueron precisados para las estaciones de primavera, verano y otoño. En invierno el intervalo fue de 45 y 55 días en T1 y T2, respectivamente. Las variables evaluadas fueron: rendimiento de forraje por corte, relación hoja/tallo (H/T), relación (hoja+tallo)/raíz (H+T)/R y el porcentaje de cobertura bajo las frecuencias planteadas. La producción de materia seca (MS) anual para T1 se incrementó. Los valores de productividad no mostraron diferencias significativas entre cultivares y entre tratamientos. Se hallaron diferencias significativas entre tratamientos para la relación H/T. Se observó interacción entre cultivares y tratamientos en la cobertura de la pastura..
\end{abstract}

Palabras clave: alfalfa, cultivar, rendimiento, frecuencia de corte, cobertura.

1.- Facultad de Ciencias Agrarias, Universidad Nacional del Litoral. Kreder 2805. (3080) Esperanza, provincia de Santa Fe, Argentina. Email: vanesaord@fca.unl.edu.ar

2.- Centro APTA Citros “Sylvio Moreira”, Instituto Agronômico, Cordeirópolis, SP, Brasil.

Manuscrito recibido el 13 de agosto de 2019 y aceptado para su publicación el 25 de noviembre de 2019.

Ordoñez, V. N.; Nescier, I. de los M.; Dovis, V. L.; Gieco, J. O.; Bonvin, C.; Ribero, G. G. \& Walker, G. A. Productividad y cobertura de cultivares de alfalfa (Medicago sativa L.) de diferentes grados de reposo invernal bajo dos frecuencias de corte. FAVE - Ciencias Agrarias 18 (1): 45-53. CC BY-NC-SA 4.0 (c) (1) () 


\section{ABSTRACT \\ Productivity and coverage of alfalfa cultivars (Medicago sativa L.) of different degrees of winter rest under two cutting frequencies .}

The objective of this work was to evaluate the forage production and the percentage of coverage of 3 alfalfa crops with different degrees of winter rest (GRI), sometimes at two cutting frequencies for a year. Three cultivars (GR6-Verzy), (GR9-Mecha) and (GR10-Ruano) were used. The experimental design was divided plots with four repetitions. Two treatments defined by the cutting intervals were established: T1: 25 days and T2: 35 days. These were specified for the spring, summer and fall seasons. In winter the interval was 45 and 55 days in T1 and T2, respectively. The variables evaluated were: forage yield per cut, leaf/stem ratio (H/T), ratio (leaf+stem)/ root $(\mathrm{H}+\mathrm{T}) / \mathrm{R}$ and the percentage of coverage under the proposed frequencies. Annual dry matter (DM) production for T1 increased. The productivity values showed no significant differences between cultivars and between treatments. Significant differences were found between treatments for the $\mathrm{H} / \mathrm{T}$ ratio. Interaction between cultivars and treat ments in pasture cover was observed.

Key words: alfalfa, cultivate, yield, cutting frequency, coverage.

\section{INTRODUCCIÓN}

En Argentina, la pastura de alfalfa (Medicago sativa L.) constituye el principal componente de la oferta forrajera para sistemas de producción bovina, de carne y leche. Se contabilizan aproximadamente 3,7 millones de hectáreas de alfalfa sembradas en un $60 \%$ como cultivos puros (1).

Su amplia difusión es debida al hecho de aportar forraje de calidad y de bajo costo, además de poseer un alto potencial de rendimiento en masa seca (MS) y adaptabilidad a diversas condiciones ambientales (suelo, clima) y de manejo (14). Romero, N. (10) sostiene que para obtener una alta cantidad y calidad de forraje, asociadas con una buena persistencia de la alfalfa, debe usarse una frecuencia de defoliación que permita a la planta producir y al mismo tiempo recuperarse del corte, esta frecuencia es considerada de aproximadamente 35 días entre cortes o pastoreos. En lo referente a calidad del forraje, no sólo se debe considerar el contenido de proteína sino también otros parámetros, como porcentajes de hoja, tallo, contenido de fibras, lignina y digestibilidad. Cortada en estados inmaduros, la alfalfa produce forraje de mayor calidad, reduciendo significativamente su producción y persistencia (11). Según Parsi et al. (8) la pérdida física de las hojas, tallos o ambos, es el principal estrés que obliga a la planta a movilizar las reservas acumuladas en la corona para producir nuevas hojas, a fin de recuperar su capacidad fotosintética. El efecto de la defoliación temprana sobre la calidad es siempre positivo, debido a que retarda el proceso de desarrollo y lignificación de las hojas en estados iniciales.

Los cultivares de diferentes GRI difieren, entre otras características, por su capacidad de crecimiento después del corte o pastoreo, encontrándose variación en la 
cantidad y tamaño de tallos que se regeneran desde la corona (12). Ponce, M. (9) afirma que cultivares sin reposo invernal (SRI) son los que poseen el mayor rendimiento y en un mayor período del año cuando son comparados con los otros grupos, en los primeros dos años de cultivo. Sin embargo, según evaluaciones realizadas en una misma localidad muchas veces no se observan diferencias entre cultivares de distinto grado de reposo invernal.

Para lograr aumento progresivo en la productividad y persistencia de los lotes de alfalfa es necesario mejorar la comprensión del efecto de las condiciones ambientales sobre los procesos fisiológicos de la planta, crecimiento, acúmulo de reservas y brotación y además, cómo las prácticas de manejo los modifican (4).

La determinación de momentos óptimos de corte tiene como objetivo mantener el equilibrio entre máxima productividad y calidad, con longevidad de la pastura. Mendoza Pedroza, S. et al. (5) sostienen que la frecuencia de corte de alfalfa debe definirse estacionalmente, teniendo en cuenta la velocidad de crecimiento de la pastura, para lograr los máximos rendimientos anuales de forraje y mantener su persistencia.

Las defoliaciones o cortes realizados en una fase temprana de desarrollo afectan la persistencia de la alfalfa, por reducir el tamaño y el vigor de la corona, dada la menor acumulación de reservas, disminuyendo concomitantemente el número y vigor de los nuevos brotes (6, 13 y 17). Mendoza Pedroza et al. (5) al evaluar rendimientos con diferentes frecuencias de corte, obtuvieron como resultado menores valores cuando se cortó la pastura cada 3 o 4 semanas, siendo el mayor intervalo de 6 o 7 semanas. Adjudicaron esta diferencia al agotamiento de reservas de carbohidratos debido al escaso tiempo de recuperación, lo que también perjudicaría la persistencia de la pastura. En concordancia, en un ensayo de Ventroni et al. (16), donde se evaluaron distintos cultivares tratados en diferentes frecuencias de corte (20, 30 y 40 días), se encontró que la supervivencia fue la menor con cortes cada 20 días.

El objetivo de este trabajo fue evaluar la producción, partición de la biomasa en diferentes órganos de la planta y el porcentaje de cobertura de 3 cultivares de alfalfa con distinto GRI, sometidas a dos frecuencias de corte.

\section{MATERIALES Y MÉTODOS}

La pastura se implantó en el campo experimental de Producción Animal de la Facultad de Ciencias Agrarias de la UNL, ubicado en la localidad "Rincón de Ávila", sobre ruta Provincial $N^{\circ} 6\left(\mathrm{~km} \mathrm{81,5),} \mathrm{a} 31^{\circ}\right.$ $20^{\prime} 08^{\prime \prime}$ latitud Sur, $60^{\circ} 50^{\prime} 48^{\prime \prime}$ longitud Oeste y a una altitud de 34 m s.n.m., en la Región Centro Norte de la Provincia de Santa Fe, Argentina. La siembra se realizó en el mes de agosto del año 2017 sobre un suelo Argiudol Típico (7). El período de cortes comprendió desde el mes de septiembre de 2018 a junio de 2019. Los parámetros de calidad del suelo mostraron valores de $\mathrm{pH}=5,88 ; \mathrm{MO} 3,61 \%, \mathrm{~N}$ total 0,18 \%, S 12,3 ppm SO4-2 y P 31,4 ppm. Dada las condiciones de fertilidad del suelo, la práctica de fertilización no se consideró necesaria. El clima de la zona presenta una temperatura media anual de $18^{\circ} \mathrm{C}$, donde la media del mes más cálido es $25^{\circ} \mathrm{C}$ y la del mes más frío $7{ }^{\circ} \mathrm{C}$ y la precipitación media anual es $1070 \mathrm{~mm}$ concentrados en el semestre que abarca desde el mes de octubre a marzo, donde se registra el $69 \%$ del total 
acumulado anualmente. Los meses estivales (febrero-marzo) presentan los mayores registros, mientras que el trimestre invernal (junio-julio-agosto) acumula los menores registros (3).

Se utilizaron tres cultivares de alfalfa, uno de reposo invernal intermedio (GR6-Verzy) y dos sin reposo invernal (GR9-Mecha) y (GR10-Ruano). El diseño experimental utilizado fue el de parcelas divididas con cuatro repeticiones (parcelas: variedades; subparcelas: frecuencias de corte). El tamaño de cada unidad experimental fue de $2 \mathrm{~m}$ de ancho por $10 \mathrm{~m}$ de largo.

Previo a la siembra se realizaron trabajos de preparación del suelo con la finalidad de lograr una correcta germinación, emergencia y alto porcentaje de establecimiento. Se utilizó una densidad de siembra de $14 \mathrm{~kg} / \mathrm{ha}$ de semilla peleteada conteniendo inoculante (Rhizobium meliloti), fungicida, insecticida y cubierta de carbonato de calcio. El espaciamiento entre hileras fue de $17 \mathrm{~cm}$.

Se establecieron 2 tratamientos definidos por los intervalos de corte: T1: 25 días y T2: 35 días. Estos fueron establecidos para las estaciones de primavera, verano y otoño. En invierno, debido a la menor tasa de acumulación de biomasa de la planta se utilizó un intervalo de corte de 45 y 55 días para la recuperación de la pastura en T1 y T2, respectivamente. En el período de ensayo se realizaron 11 cortes para $\mathrm{T} 1$ y 8 cortes para T2.

Las variables evaluadas fueron:

Rendimiento de forraje por corte: se determinó en cuadros de $0,25 \mathrm{~m}^{2}$ por parcela a una altura de corte de $8 \mathrm{~cm}$. Las muestras fueron cortadas manualmente con tijera y pesadas para obtención de la masa fresca. Posteriormente, fueron secadas en estufa con corriente de aire forzado a $55{ }^{\circ} \mathrm{C}$ hasta masa constante. Luego se determinó el porcentaje de materia seca (\%MS). El rendimiento de forraje expresado en toneladas de MS por hectárea (tn MS hä).

Para la determinación de las relaciones $H / T$ y $(H+T) / R$ : se realizó el muestreo tomando 3 plantas al azar por parcela, a una profundidad de $15 \mathrm{~cm}$, sobre $30 \mathrm{~cm}$ de la línea de siembra. Las muestras se secaron en estufa con corriente de aire forzado a 55 ${ }^{\circ} \mathrm{C}$ hasta masa constante, posteriormente fueron separadas y pesadas cada fracción de planta (hoja, tallo y raíz), para así determinar las proporciones correspondientes.

Porcentaje de cobertura: se registró a través de la observación de espacios vacíos en un metro lineal de la línea de siembra, con tres repeticiones por parcela, para el cálculo de porcentaje de cobertura, realizándose las mediciones al final del período de ensayo, según los tratamientos T1 y T2.

Los resultados se analizaron con uso del programa Infostat (2) por medio de análisis de Varianza factorial con interacción (ANAVA) con dos fuentes de variación (frecuencias de corte y cultivares), cuando el modelo detectó diferencias significativas, estas fueron sometidas a Test de Tukey $\mathrm{p}<0,05$.

\section{RESULTADOS Y DISCUSIÓN}

No se observaron diferencias significativas en la producción acumulada anual de MS entre cultivares y entre tratamientos (Fig. 1). El rendimiento acumulado resultó 21,3 tn MS ha- ${ }^{-1}$ para T1 y 18,8 tn MS ha- ${ }^{-1}$ para T2, para el cultivar Mecha, de 18,6 tn MS ha-1 en T1 y T2, para el cultivar Ruano y 19,2 tn MS ha- ${ }^{-1}$ en T1 y 20,7 tn MS ha ${ }^{-1}$ en T2, para el cultivar Verzy (Fig. 1). 


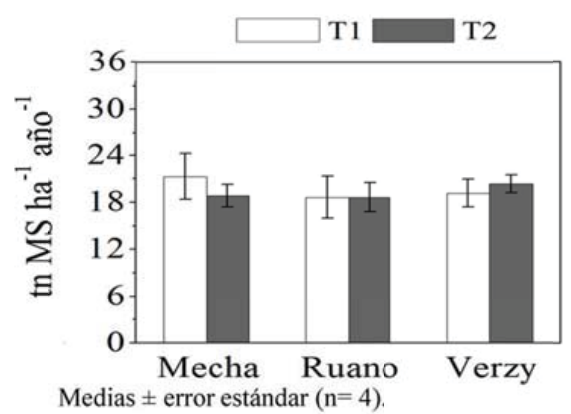

Figura 1. Producción en cultivares de alfalfa de diferente grado de reposo invernal, sometidos a dos frecuencias de corte: 25 días entre cortes (T1) y 35 días entre cortes (T2).

Se observaron diferencias significativas entre tratamientos para la relación $\mathrm{H} / \mathrm{T}$ y en la relación (H+T)/R (Fig. 2), no así entre los cultivares que tuvieron un comportamiento similar y sin diferencias. Las plantas sometidas a T1 tuvieron una mayor proporción de $\mathrm{H} / \mathrm{T}$ respecto de $\mathrm{T} 2$, de lo que se infiere que puedan tener mejor calidad y mayor digestibilidad, debido a la mayor proporción de hoja respecto del tallo que tiene un alto contenido de fibras y menor digestibilidad. $\mathrm{Al}$ respecto para Romero et al. (11) un forraje de alta calidad está relacionado con un alto contenido de hojas y bajo porcentaje de tallos, disminuyendo la digestibilidad del forraje con la madurez.

Los cortes frecuentes producen un aumento en la calidad nutritiva del forraje, no obstante Valentine, L. et al. (15) y Villegas, A. et al. (17) observaron que las plantas tienden a desaparecer por el agotamiento de las reservas de carbohidratos, ocasionando un lento rebrote de la pastura. Esto también se ve reflejado en la mayor proporción de fracción aérea con respecto a la cantidad de raíces $(H+T) / R$. Se puede vincular esta relación con una incompleta reposición de reservas en detrimento del crecimiento radicular, lo que puede comprometer la persistencia de las plantas a lo largo del tiempo, y así la cobertura de la pastura.

Al analizar el porcentaje de cobertura después de un año de cortes, se observó sólo interacción entre cultivares y tratamientos de frecuencia de corte. Hallándose que independientemente de su GRI, la menor frecuencia, entre cortes, afectó negativamente a los cultivares Verzy y con mayor intensidad al cultivar Mecha. (Fig. 2). Al respecto, Ventroni et al. (16) encontraron que el efecto de la frecuencia de la defoliación sobre la biomasa aérea y supervivencia es independiente del GRI y que el manejo de la defoliación es de mayor importancia comparado con la elección del GRI. 


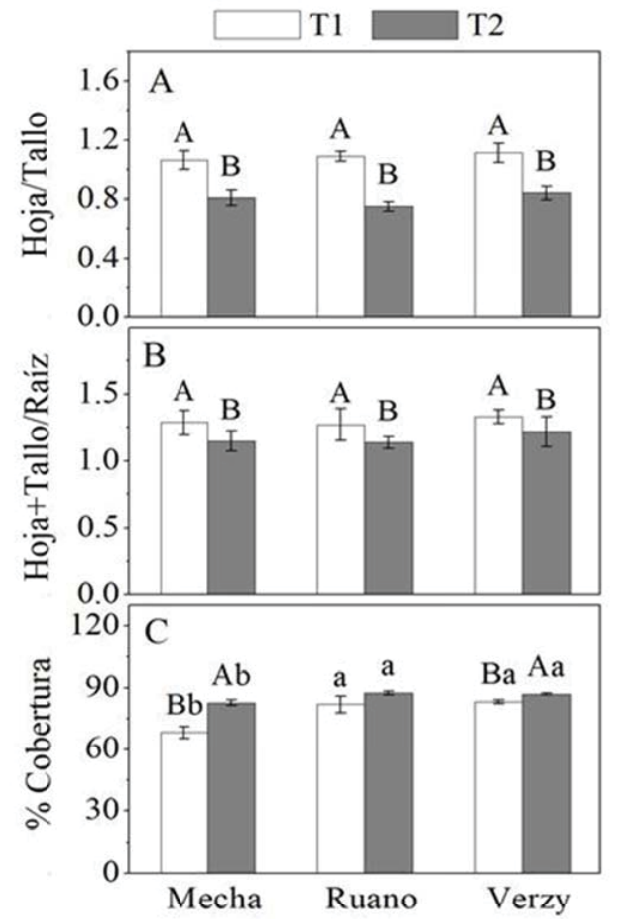

Letras mayúsculas diferentes indican diferencias estadisticas para cada cultivar y letras minúsculas indican diferencias entre los cultivares dentro de una misma frecuencia de corte. Media \pm error estándar $(n=4$ Test de Tukey para $\mathrm{p}<0,05$ ).

Figura 2. Proporción Hoja/Tallo (A); Relación Hoja+Tallo/Raíz (B) y porcentaje de cobertura después de un año de cortes (C), en cultivares de alfalfa de diferente grado de reposo invernal, sometidos a dos frecuencias de corte: 25 días entre cortes (T1) y 35 días entre cortes (T2).

Al analizar el comportamiento estacional de los cultivares (Fig. 3) se observó, en los primeros cortes, que la relación ( $\mathrm{H}^{+-}$ $\mathrm{T}) / \mathrm{R}$ se redujo gradualmente conforme el avance del desarrollo de la planta. No se observaron diferencias en $\mathrm{T} 1$ y $\mathrm{T} 2$ entre los cortes, al mismo tiempo se registró una reducción en el stand de plantas para T1.

En los primeros cortes, durante primavera e inicio del verano no hubo diferencias en la producción entre las dos frecuencias de corte (Fig. 4). A partir de diciembre o enero se registró una reducción en la producción por corte, de esta forma resulta viable durante la primavera establecer una frecuencia de corte de 25 días. A partir del mes de diciembre sería recomendable espaciar la frecuencia de corte a 35 días con el objetivo de mantener el stand de plantas y la productividad. 


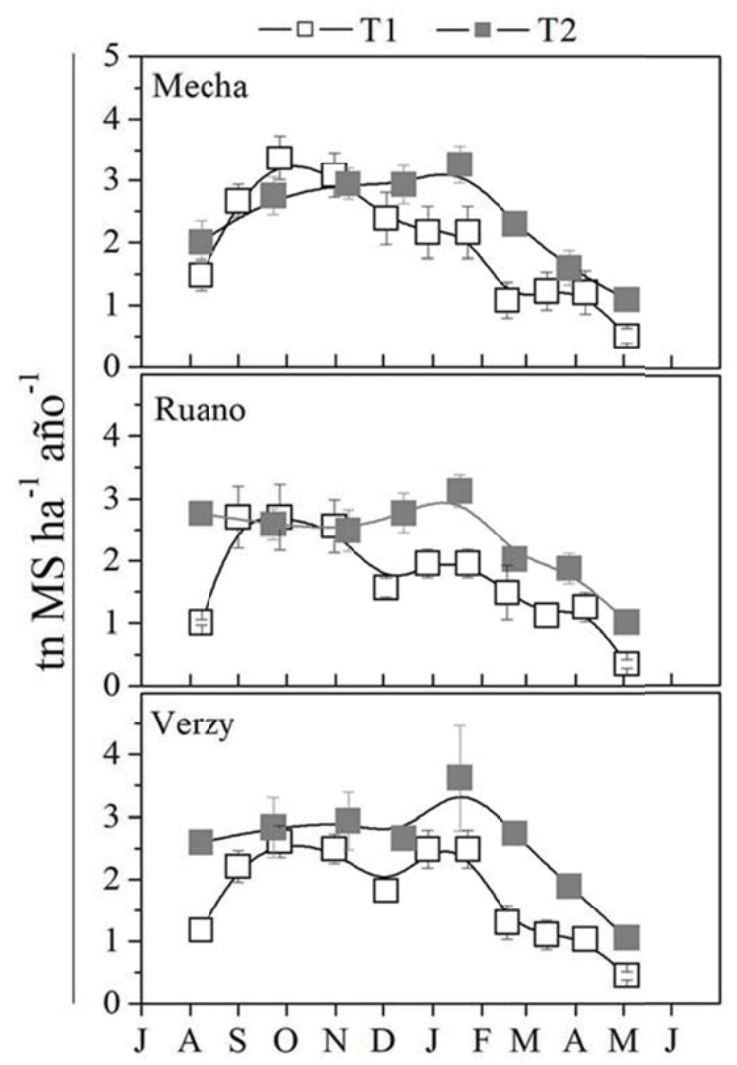

Figura 3. Variación estacional (abscisas: meses del año) de relación Hoja/Tallo y de la relación (Hoja+Tallo)/Raíz en tres cultivares de alfalfa de diferente grado de reposo invernal, sometidos a dos frecuencias de corte: 25 días entre cortes (T1) y 35 días entre cortes (T2). Media \pm error estándar $(n=4)$. 


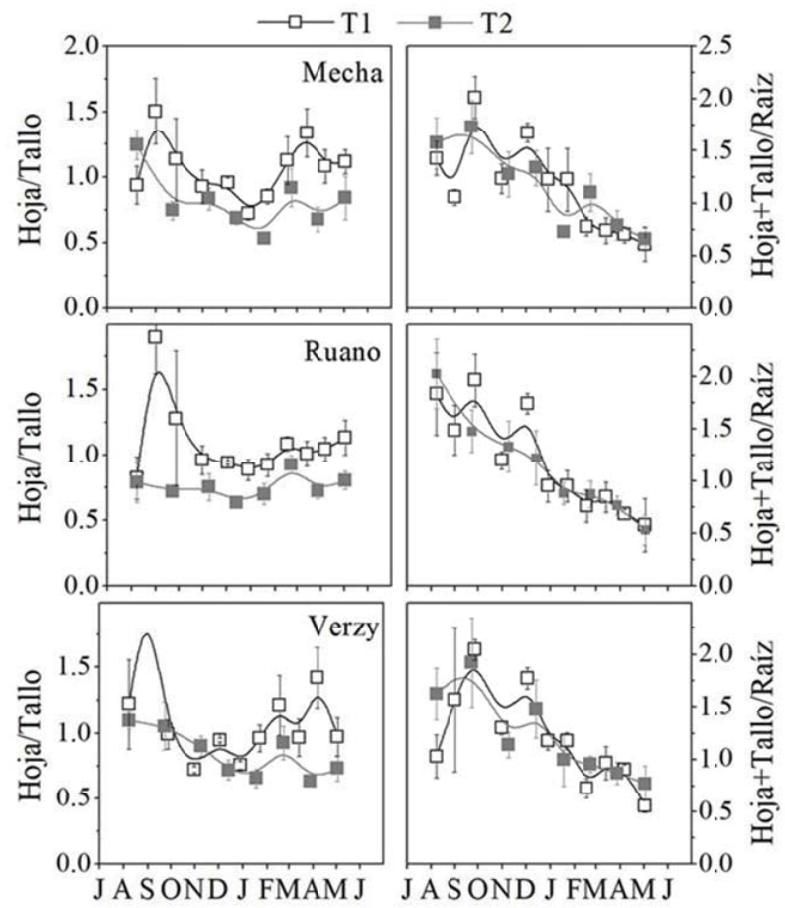

Figura 4. Variación estacional de la producción de materia seca en tres cultivares de alfalfa de diferente grado de reposo invernal, sometidos a dos frecuencias de corte: 25 días entre cortes (T1) y 35 días entre cortes (T2). Media \pm error estándar $(n=4)$.

\section{CONCLUSIONES}

La producción de MS anual para T1 se incrementó debido a la mayor cantidad de cortes. El establecimiento de una frecuencia fija de corte no parece ser la estrategia más apropiada para los cultivares estudiados. El cultivar Mecha tuvo una menor cobertura. Una combinación de frecuencias de corte, que considere las variaciones en las condiciones determinantes del crecimiento y la respuesta de la pastura, debería ser considerada.

\section{AGRADECIMIENTOS}

Esta investigación fue financiada por un proyecto CAI+D UNL, convocatoria 2016.

\section{BIBLIOGRAFÍA}

1.- BASIGALUP, D. H. 2014. Situación de la alfalfa en Argentina. $5^{\circ}$ Jornada Nacional de Forrajes Conservados. Ediciones INTA. pp. 95-99.

2.- DI RIENZO, J. A.; CASANOVES, F.; BALZARINI, M. G.; GONZALEZ, L.; TABLADA, M. Y ROBLEDO, C. W. InfoStat versión 2016. Grupo InfoStat, FCA, Universidad Nacional de Córdoba, Argentina. 
3.- INTA RAFAELA. Agrometeorología Rafaela en https://inta.gob.ar/paginas/agrometeorología-rafaela (visita 13 de agosto de 2019). Esperanza: EEA INTA, 2018.

4.- FICK, G. W.; HOLT, D. A. Y LUGG, D. G. 1988. Environmental physiology and crop growth. In: A. A. Hanson, D. K. Barnes and R.R. Hill (eds.) Alfalfa and alfalfa improvement.

5.- MENDOZA PEDROZA, S. I.; HERNANDEZ GARAY, A.; PEREZ PEREZ, J.; QUERO CARRILLO, A. R.; ESCALANTE ESTRADA, A. S.; ZARAGOZA RAMIREZ, J. L. Y RAMIREZ REYNOSO, O. 2010. Respuesta productiva de la alfalfa a diferentes frecuencias de corte. Rev Mex Cienc Pecu; 1(3):287-296.

6.- MUSLERA, P. E. Y RATERA, G. C. 1984. Praderas y forrajes. Producción y aprovechamiento. Editorial Mundi Prensa. Madrid. España. 702p.

7.- PANIGATTI, J. 2010. 200 años, 200 suelos. Buenos Aires: INTA.

8.- PARSI, J.; GODIO, L.; MIAZZO, R.; MAFFIOLI, R.; ECHEVARRÍA, A. Y PROVENSAL, P. 2001. Valoración nutritiva de los alimentos y formulación de dietas. Cursos de Producción Animal, FAV, UNRC. www.produccion-animal.com.ar

9.- PONCE, M. 2011. Producción de forraje: Estado de desarrollo de alfalfas con distinto grado de reposo. Eduvin. Córdoba, Argentina. 77 p.

10.- ROMERO, N. 2011. Efecto del período de pastoreo y estado de madurez sobre la producción y persistencia de alfalfas con distintos grados de dormancia. Ediciones INTA EEA. INTA Anguil Ing. Agr. Guillermo Covas. pp 1-20.

11.- ROMERO, N. A.; COMERÓN, E. A. Y USTARROZ, E. 1995. Manejo y utilización de la alfalfa. La Alfalfa en la Argentina, INTA Cuyo, 150-170.

12.- ROSSANIGO, R; SPADA, M. Y BRUNO, O. 1995. Evaluación de cultivares de alfalfa y panorama varietal en la Argentina. En: Hijano, E. y Navarro, A. La alfalfa en la Argentina. Buenos Aires: INTA C.R. Cuyo. Cap. 4. p 63-78. (ISSN: 0327-3377).

13.- TEIXEIRA, E. I.; MOOT, D. $y$ BROWN, H. E. 2008. Defoliation frequency and season affected radiation use efficiency and dry matter partitioning to roots of lucerne (Medicago sativa L.) crops. Eur. J. Agron. 28:103-111.

14.- TOLISSO, J. L.; DALLA FONTANA, L. A.; QUIÑONEZ, A. G.; MOLLO, A. J. Y BOGLIONE, G. A. 1997. Evaluación de cultivares de alfalfa bajo condiciones de pastoreo. FAVE: vol 10. $\mathrm{N}^{\circ}$ 1- 2. 1997.

15.- VALENTINE, L. Y MATTHEW, C. 1999. Plant growth, development and yield. In: White, J., Hodgson, J. editors. N Z Pasture Crop Sci. Auckland, Nez Zeland: Oxford University Press: pp 1127.

16.- VENTRONI, L.; VOLENEC, J. J. Y CANGIANO, C. A. 2010. Interacción frecuencia de corte y grado de reposo en la productividad y supervivencia de alfalfa. Facultad de Ciencias. Agrarias-UNMdP. Purdue University, EEUU. INTA EEA, Balcarce. Revista Argentina de Producción Animal Vol. 30 Supl. 1:203-431.

17.- VILLEGAS, A. Y.; HERNÁNDEZ, G. A.; MARTÍNEZ, H. P. A.; PÉREZ, P. J.; HERRERA, H. J. G. Y LÓPEZ, C. C. 2006. Rendimiento de forraje de variedades de alfalfa en dos calendarios de corte. Rev Fitotecnia Mex; 29 (4):369 -372 . 